\title{
Candida Pneumonia: What We Know and What We Don't
}

\author{
Christina E. Barkauskas, MD, and John R. Perfect, MD
}

\author{
Corresponding author \\ Christina E. Barkauskas, MD \\ Department of Medicine, Division of Pulmonary and Critical Care \\ Medicine, Duke University Medical Center, Erwin Road, Box 3182, \\ Durham, NC 27710, USA. \\ E-mail: barka001@mc.duke.edu
}

Current Fungal Infection Reports 2009, 3:21-31

Current Medicine Group LLC ISSN 1936-3761

Copyright (C) 2009 by Current Medicine Group LLC

The diagnosis of Candida pneumonia is a difficult one to make, and some experts often question whether the entity truly exists. This article assimilates the information currently available regarding Candida pulmonary infection to elucidate the presentation of the disease and the hosts in which it occurs. It also describes how to interpret the presence of Candida in respiratory secretions from both immunocompetent and immunocompromised patients. Finally, it describes how the diagnosis of Candida pneumonia can be made, which patients with suspected disease should be treated, and which antifungal agents should be used.

\section{Introduction}

Many experts often question whether Candida pneumonia truly exists. The topic of Candida pneumonia has been declared an "evidence-based-free zone" by many in the infectious disease community. In this article, we attempt to assimilate the current available information regarding Candida pulmonary infection to give a sense of where the evidence lies, where it is lacking, and what conclusions and recommendations we can make regarding suspicion of the disease, diagnosis of the disease, and management of the disease in both immunocompetent and immunosuppressed patients.

The History of Candida Pulmonary Infection The diagnosis of Candida pneumonia has always been difficult, and clinicians have struggled for years with the ability to definitively recognize cases of such. Some argue that this is because the entity is extremely rare; others argue that this is because effective diagnostic tools are lacking. Whatever the reason, it is difficult to generate a clinical case series to study this question, and as a result, most clinical case studies describing Candida pneumonia are based on autopsy studies.

\section{The Spectrum of Candida Pulmonary Infection:} Lessons From Autopsy Studies

A variety of autopsy studies aiming to better define Candida pneumonia have been reported in the literature over the past three decades. The most notable of these are described below and are summarized in Table 1.

In 1977, Masur et al. [1] published a series of 30 patients with histologic evidence of Candida pulmonary infection (ie, parenchymal invasion) that was identified after review of 995 consecutive autopsies from 1973 to 1974 at Memorial Sloan-Kettering Cancer Center and New York Hospital. This evidence suggested an incidence of Candida pneumonia in this population of 3\% [1]. Twenty-eight of the 30 patients had underlying neoplastic disease; 24 of these 28 patients had neutropenia (absolute neutrophil count $[\mathrm{ANC}]<500)$ for greater than 10 days. Interestingly, significant correlation between postmortem lung culture and histologic evidence of invasive candidiasis was lacking. In the 30 cases of invasive Candida pulmonary infection described by Masur et al. [1], only 18 lung cultures were positive for Candida. Similarly, of the 995 autopsies studied by Masur et al. [1], 61 resulted in postmortem pulmonary cultures positive for Candida; in only three of these 61 cases was histology positive for invasive disease.

Upon studying the distribution of organisms to the lungs in their autopsy series, Masur et al. [1] set out to characterize the route by which Candida spread to the lung. It was suggested that there are two sources of Candida pneumonia: 1) hematogenous dissemination, characterized by an absence of bronchial spread and presence of Candida pseudohyphae found growing from within pulmonary vessels into surrounding lung tissue; and 2) endobronchial dissemination, characterized by intrabronchial and alveolar changes associated with aspiration in many cases. Cases of Candida pneumonia caused by hematogenous dissemination were often characterized by finely nodular, diffuse 


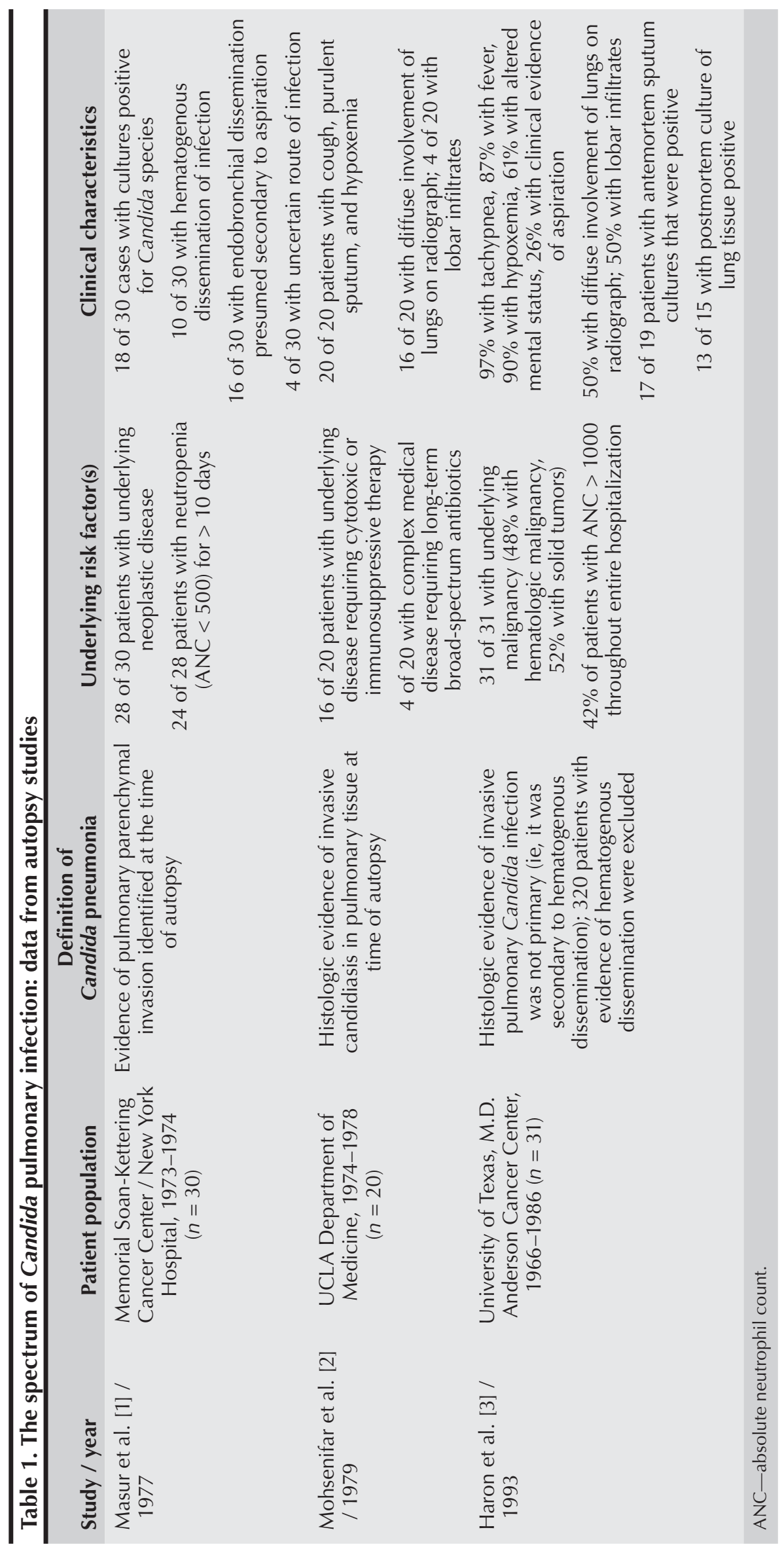


infiltrates and occasionally necrotizing pneumonias and acute pulmonary mycetoma. Cases of Candida pneumonia caused by endobronchial dissemination were often characterized by evidence of local or diffuse bronchopneumonia on imaging. Of the 30 patients described by Masur et al. [1], 10 were suspected to have had hematogenous spread of infection to the lungs, 16 were suspected to have had infection spread to the lungs by way of aspiration, and four patients had uncertain routes of infection. Although the specific primary sources of hematogenous dissemination of Candida were not described in this study, one can hypothesize that these can include anything from intraabdominal abscess, to right-sided endocarditis with septic emboli occurring in the setting of intravenous drug abuse, to septic thrombophlebitis occurring in the setting of contaminated central venous catheters.

From Masur et al.'s [1] data, it appears that Candida pneumonia is a rare phenomenon that typically occurs in the setting of severe underlying malignant disease in patients who develop severe sepsis and/or multiorgan system failure. The caveat to this description is the fact that the data were collected predominantly from a referral center that specializes in the diagnosis and treatment of hematologic/oncologic disorders. It is certainly the case that referral bias could have skewed these results.

The next prominent autopsy series was published by Mohsenifar et al. [2] in 1979. This was a retrospective study of 20 cases of pulmonary candidiasis diagnosed at autopsy between 1974 and 1978 in the Department of Medicine at the UCLA School of Medicine. As was the case in the series by Masur et al. [1], patients were included only if there was histologic evidence of invasive candidiasis in pulmonary tissue; they were excluded if postmortem culture of lung tissue revealed growth of Candida but histologic evidence of invasion was lacking. Of these 20 cases, all patients clinically displayed cough with purulent sputum production and hypoxemia; four patients had occasional hemoptysis. Sixteen of the 20 patients had serious underlying diseases requiring immunosuppression (ie, steroids) or cytotoxic therapy (ie, chemotherapy). The remaining four patients had complicated medical disease requiring prolonged courses of broad-spectrum antibiotics. Fourteen of the 20 patients had low leukocyte counts, 10 of the 20 patients had an underlying malignant condition, 16 of the 20 patients had diffuse involvement of the lungs on radiograph, and four patients had lobar infiltrates.

This study was consistent with that published by Masur et al. [1], suggesting again that the vast majority of cases of invasive Candida pulmonary infection occur in patients who are significantly immunosuppressed. Contrary to Masur et al.'s [1] data, however, it appears that these cases do not universally occur in patients with underlying malignant conditions. In fact, patients receiving high-dose steroids for nonmalignant conditions and patients with complicated medical illness requiring pro-

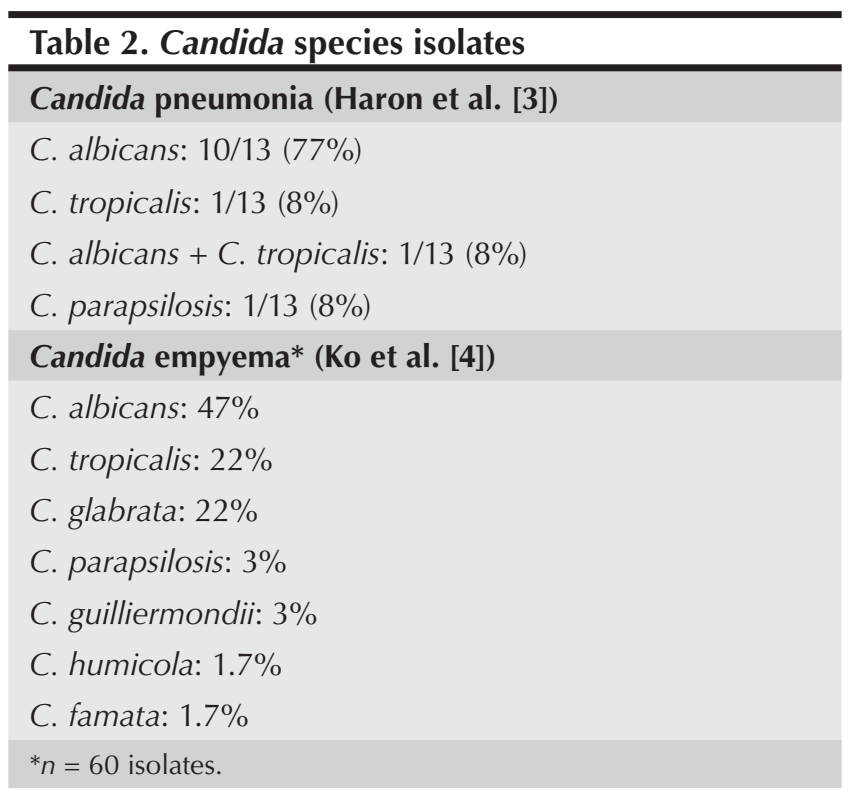

longed courses of broad-spectrum antibiotics seem to be at risk as well.

The final large autopsy series was published by Haron et al. [3] and described a series of 31 patients treated at the University of Texas, M.D. Anderson Cancer Center between 1966 and 1986 who were found at autopsy to have primary Candida pneumonia. The authors reviewed a total of 7725 autopsies, from which there were 351 cases of autopsy-proven invasive pulmonary Candida infection (incidence of $4.5 \%$ ). It is important to note that, unlike the previous autopsy studies, the authors of this study excluded 320 patients with evidence of invasive pulmonary Candida infection that was not primary (ie, it was secondary to hematogenous dissemination). All 31 patients with primary Candida pneumonia had underlying malignancy $(48 \%$ with hematologic malignancy, $52 \%$ with solid tumors). Thirteen patients $(42 \%)$ had adequate neurophil counts (ANC $>1000)$ throughout their hospitalizations; nine patients $(29 \%)$ had severe neutropenia (ANC < 100). The majority of these patients presented with dyspnea and/or tachypnea $(97 \%)$, fever $(87 \%)$, and hypoxemia $(90 \%)$. Sixty-one percent of patients displayed altered mental status and there was clinical evidence of aspiration in $26 \%$ of patients. Seven patients $(23 \%)$ were found to have Candida esophagitis. Approximately half of the patients included in the series had diffuse bilateral pulmonary infiltrates, whereas the other half had infiltrates limited to one or more lobes.

Antemortem sputum culture was positive in 17 of 19 patients. Postmortem culture of lung tissue was obtained from 15 patients, 13 of which were positive (10 Candida albicans, 1 C. tropicalis, 1 C. albicans and C. tropicalis, 1 C. parapsilosis) (Table 2). Histopathologic findings were most commonly bronchopneumonia (81\%); intra-alveolar exudates $(52 \%)$ and intra-alveolar hemorrhage $(52 \%)$ were also common. Evidence of aspirated material was found in 
$10 \%$ of cases. Only one patient displayed evidence of intravascular involvement. Upon review of all data, the death of $84 \%$ of these patients (26 of 31 ) was felt to have been secondary to primary Candida pneumonia $(n=21)$ or to concomitant Candida and bacterial pneumonia $(n=5)$.

As was the case with the series by Masur et al. [1], this series also included patients from a specialized referral center, skewing the population to include only those patients with an underlying malignancy. A substantial fraction of these patients had clinical evidence of aspiration prior to death, although, unlike in the study by Masur et al. [1], this was confirmed by histology in only a fraction of cases.

What can we gather from these autopsy studies? Candida pulmonary infection, whether primary or secondary, does seem to exist in certain distinctly immunocompromised populations. Most patients with postmortem evidence of invasive pulmonary disease had antemortem clinical evidence of pneumonia, characterized by pulmonary infiltrates, fever, and hypoxemia. This diagnosis is rare (with an incidence of $3 \%-4.5 \%$ based upon previous studies $[1,3])$ and seems to occur predominantly in patients with underlying malignancy, although it can also occur in patients who are immunosuppressed for other reasons.

In considering these data, we realize that the groups from which these data were obtained are not necessarily representative of everyone in an inpatient hospital setting. Further, given advancements in the diagnosis and treatment of malignant and rheumatologic conditions with cytotoxic and/or immunosuppressive therapy, and given the increase in the number of patients who have undergone solid organ transplantation, the populations of patients included in these studies are not necessarily representative of the population we care for today.

\section{Other Clinical Presentations of Candida Pulmonary Infection}

Candida pulmonary infection is not necessarily limited to pneumonia. Alternative presentations of this disease, including Candida empyema and a variety of postsurgical Candida infections in lung transplant patients, are described below.

\section{Candida empyema: fungal empyema thoracis}

As reported by Ko et al. [4], the incidence of fungal empyema thoracis has increased in recent years. This entity is defined by the following criteria: 1) the presence of an exudative pleural effusion with isolation of fungal species, 2) the presence of significant signs of infection, and 3) repeat isolation of fungal species from the pleural effusion or the isolation of fungal species from other specimens such as blood or sputum. Of the 67 patients described by Ko et al. [4], 90\% had underlying medical disease and $84 \%$ developed nosocomial infection (most acquired in the intensive care unit). Regarding the microbiology of these infections, $82 \%$ of the clinically significant isolates were Candida species $(47 \%$ C. albicans, $22 \%$ C. tropicalis, $22 \%$ C. glabrata, $3 \%$ C. parapsilosis, $3 \%$ C. guilliermondii, $1.7 \%$ C. humicola, and $1.7 \%$ C. famata) (Table 2). Most of the patients with fungal empyema thoracis received systemic antifungal therapy $(73 \%), 57 \%$ underwent closed drainage therapy, $16 \%$ required pleural irrigation, and $9 \%$ required decortication. The crude mortality, despite these therapies, was $73 \%$. Through multivariate analysis, it was shown that patients with an underlying immunocompromised state and patients with respiratory failure had an increased risk of death (RR of 1.58; $P<0.005$ and RR of $2.31 ; P<0.001$, respectively).

\section{Spectrum of Candida pulmonary infections in lung transplant recipients}

Candida species have also been implicated in pulmonary infections in patients who have undergone lung transplantation. As lung transplant is becoming a more common therapeutic option for patients with end-stage lung disease, these infectious complications must be considered by the providers caring for these patients.

The spectrum of post-lung transplant pulmonary complications secondary to Candida is quite broad. Kanj et al. [5], in a review of a series of patients undergoing lung and heart-lung transplantation from 1992 to 1995 who later developed fungal infection, reported three different manifestations of Candida pulmonary infection.

First, disease can be manifest by Candida pneumonia, with resulting disseminated disease. This particular manifestation was described in a patient who, on postoperative day 5 , in the setting of profound respiratory failure requiring extracorporeal membrane oxygenation, was found to have pulmonary hemorrhage and focal necrosis in the left lung with no rejection; culture of lung tissue grew C. albicans. The patient was treated with fluconazole but ultimately developed cultures positive for C. albicans from blood, sputum, bronchoalveolar lavage (BAL), and peritoneal fluid. Despite therapy being switched to amphotericin $\mathrm{B}$, the extent of the patient's infection worsened and the patient ultimately died.

Next, Candida infection in the post-lung transplant setting can take the form of disseminated candidiasis originating from the donor lung. This manifestation was described in a patient who, on postoperative day 5, underwent cardiopulmonary arrest with follow-up bronchoscopy yielding a BAL culture positive for C. albicans. The patient was treated initially with vancomycin and ceftazidime; because of persistent fever and leukocytosis, fluconazole was started on postoperative day 7. Subsequent cultures from blood and peritoneal fluid were positive for C. albicans. Karyotypic analysis revealed that the C. albicans grown from the transplant recipient's cultures matched the C. albicans that was isolated from the donor lung prior to transplant. As the donor was on mechanical ventilation for only 1 day prior to death, it was suspected that the donor lungs were simply 
colonized with Candida. In the setting of profound immunosuppression in the recipient, the presence of this Candida was enough to set up a rapidly evolving pneumonia.

The final manifestation of Candida pulmonary infection that has been described in patients after lung transplant is infection and subsequent poor healing of the bronchial anastomosis sites. The patient described by Kanj et al. [5] was a patient with an uncomplicated postoperative course who presented on postoperative day 11 with dyspnea on exertion and bilateral pulmonary infiltrates. Bronchoscopy revealed stenotic bronchial anastomoses with erythematous mucosa and possible tissue necrosis. Endobronchial biopsy revealed coagulation necrosis with fungal forms; BAL cultures grew C. albicans. This patient was initially treated with both intravenous and inhaled amphotericin B. After discharge, the patient completed a 4-month course of fluconazole and once-weekly nebulized amphotericin B, with ultimate resolution of the abnormalities at her anastomoses.

\section{Clinical Manifestations of Disease:}

\section{How Severe Is Candida Pulmonary Infection?}

The previously described autopsy studies are valuable in that they allow for characterization of Candida pulmonary infection (ie, who gets it, what it looks like) based upon strict histologic criteria. The pitfall of these studies, however, is that it is impossible to determine a true mortality rate for Candida pulmonary infection given the fact that the mortality rate of the patients included in these autopsy studies is $100 \%$.

What is the mortality rate associated with Candida pulmonary infection? This question is difficult, if not impossible, to answer given the broad spectrum of disease and the lack of clarity in making a definitive diagnosis. Chen et al. [6], however, in a report of a series of invasive fungal infections, attempted to characterize patient outcomes and prognostic factors.

Chen et al.'s [6] study reviewed all cases of pulmonary fungal infection from January 1988 to December 1997 from a university-affiliated tertiary medical center in Taiwan. In this series, acute invasive fungal infection was defined as "patients with rapid progression of disease clinically or radiographically, and at least one of the following: 1) fungal invasion of lung parenchyma noted by pathologic examination or 2) fungal isolation from sterile site such as blood or pleural effusion." Of 140 patients with pulmonary fungal infection included in the study, 20 patients (14\%) had evidence of infection with Candida species (9 C. albicans, 4 C. tropicalis, 4 C. glabrata, and 2 C. parapsilosis). Of these 20 cases of invasive Candida infection, 12 were cases of Candida pneumonia and eight were cases of systemic disease (ie, presence of pneumonia plus fungemia). The mortality rate for these patients was $70 \%$ (compared with $36.4 \%$ for all 140 patients included in the series). Although it was suggested that invasive Candida infection is not as common as infection by Aspergillus species or Cryptococcus species, it did appear that Candida infection is associated with a significantly higher mortality rate.

Chen et al. [6] not only demonstrated the severity of Candida pulmonary infection, but also demonstrated an increase in the frequency of the disease. Although they reported five, eight, and six cases identified in 1988, 1989, and 1990, respectively, there were more than 15 cases identified annually from 1994 to 1997. This finding was corroborated by Schwesinger et al. [7] in their case series describing candidiasis and aspergillosis identified on autopsy from 1994 to 2003 . A total of 2027 autopsies were performed at a mid-sized hospital and several outlying regional hospitals in Germany. The population included in this study was more general than those populations included in Masur et al.'s [1] and Haron et al.'s [3] studies (which included a disproportionate number of severely immunosuppressed patients.) The rate of candidiasis increased dramatically from the years 1994 to 1998 to the years 1999 to $2003(P<0.0001)$. During the period from 1994 to $1998,4.4 \%$ of autopsies revealed candidiasis and $42 \%$ of these had lung/trachea involvement. During the period from 1999 to 2003, 10.4\% of autopsies revealed candidiasis (or candidiasis plus aspergillosis) and $37.5 \%$ of these had lung/trachea involvement. This suggests an incidence of Candida pneumonia in a general hospitalized population of $1.8 \%$ (from 1994-1998) that increased to $3.9 \%$ (from 1999-2003).

\section{What is the etiology of the increased incidence of invasive candidiasis?}

There is no clear-cut answer to this question; however, it is appealing to suggest that this is because we are caring for a sicker population of patients. Because of advances in many aspects of medical care, we are not only caring for a group of patients with a greater burden of underlying disease, but we are also keeping these patients alive for longer periods of time. We care for a population of patients with more advanced malignancy, often requiring aggressive chemotherapy regimens, leading to depressed neutrophil count or function. We care for a population with an increasing rate of solid organ transplants, leading to increased use of aggressive immunosuppression, which, in turn, leads to impaired T-lymphocyte function. Given the prominent role that T-lymphocytes play in immune defense against Candida (evidenced by the increased incidence of chronic candidal infection in patients with $\mathrm{T}$ lymphocyte deficiency states, such as DiGeorge syndrome or AIDS), impaired T-lymphocyte function can lead to an increased risk of candidiasis. Finally, we care for a population of critically ill patients that is often treated aggressively with broad-spectrum antibiotics, potentially leading to changes in a patient's normal flora, with poten- 
tial for overgrowth of Candida in the gastrointestinal tract. We are able to keep these complicated patients alive longer now than 10 or 20 years ago, thereby allowing increased opportunity for the establishment of invasive Candida infection.

Although the incidence of Candida pneumonia is on the rise, it is a diagnosis that might often be missed In a comparison of premortem clinical diagnoses in critically ill patients and subsequent autopsy findings in 100 patients who died in a mobile intensive care unit in 1996, Roosen et al. [8] determined that the diagnosis of 16 cases of fungal pneumonia was missed; five of these cases were felt to represent "major missed diagnoses" (ie, diagnoses that if known before death "would have led to a change in management with potential for cure or prolonged survival"). It is important to note that the precise etiology of these fungal pneumonias (ie, Candida vs other fungi) was not described in the study. These five "major missed" cases all occurred in patients who were immunosuppressed (or functionally immunosuppressed): two patients with chronic obstructive pulmonary disease on chronic corticosteroid therapy, one patient after lung transplant, one patient with decompensated liver cirrhosis, and one patient after recent chemotherapy for non-Hodgkin's lymphoma. Further, this study demonstrated that $31 \%$ of fungal infections in immunosuppressed patients were missed, despite a high index of suspicion and an aggressive diagnostic approach.

\section{Candida Pneumonia Is a Severe Disease:} How Do We Make the Diagnosis?

It is clear that Candida pneumonia, when it occurs, is a devastating disease with increasing incidence, but how do we make the diagnosis? The autopsy data described here are not robust enough to clearly inform us of how culture data from respiratory tract specimens should be interpreted. What, then, should we make of respiratory tract cultures that are positive for Candida species?

\section{There are no specific criteria used in practice to} diagnose Candida pulmonary infection

Perhaps some of the lack of clarity in how to handle the presence of Candida in a respiratory tract sample is rooted in the fact that there is no precise set of criteria for interpretation of respiratory tract samples with Candida species.

At the present time, respiratory tract samples with Candida species are most often obtained from mechanically ventilated patients. With this in mind, before attempting to determine whether the presence of Candida species in these patients represents colonization or pneumonia, it is important to consider the current characterization of ventilator-associated pneumonia (VAP).
There is no standard set of criteria for the diagnosis of VAP, but it is generally recognized as the development of a clinical pneumonia (new or progressive pulmonary infiltrates, fever, leukocytosis, or purulent tracheobronchial secretions) that has developed more than 48 hours after the initiation of mechanical ventilation [9]. Given the lack of concrete criteria for use in the definition/diagnosis of VAP, accurate epidemiologic data are limited; in most reports, however, VAP frequency is typically said to be between $8 \%$ and $28 \%$ [10].

The bacteriologic strategy to diagnose pneumonia and identify pathogens in VAP often relies upon use of bronchoscopy with BAL or protected specimen brush (PSB). BAL studies have typically relied upon a diagnostic threshold of $10^{4}$ or $10^{5}$ colony-forming units (CFU)/mL of bacteria; PSB studies have typically relied upon a diagnostic threshold of $10^{3} \mathrm{CFU} / \mathrm{mL}$ [11]. It is important to note that there is no similar threshold established for diagnosis of Candida pulmonary infection.

The most common etiologic microorganisms, as documented in a combination of 24 studies [10], are gram-negative bacilli, which account for $58 \%$ of recovered organisms. Pseudomonas aeruginosa is the most commonly recovered microorganism (24.4\%), whereas Enterobacteriaceae (including Klebsiella, Escherichia coli, Proteus, Enterobacter, and Serratia) are next most commonly recovered microorganisms (14.1\%); Staphylococcus aureus accounts for $20.4 \%$ of recovered microorganisms. Fungi (not further characterized in these studies, but presumably including Candida species) constitute only $0.9 \%$ of all recovered microorganisms suspected to be the etiologic agent responsible for VAP. (Note that these estimates are based on studies that do not necessarily include rigorous histologic analysis of tissue, perhaps accounting for the somewhat lower incidence of fungal VAP reported here as compared with that described in some of the previously mentioned studies.)

\section{Defining Candida colonization versus infection in immunocompetent patients}

In order to better determine the significance of positive pulmonary cultures when histologic data are not available, investigators have sought to better characterize Candida colonization versus infection in immunocompetent patients.

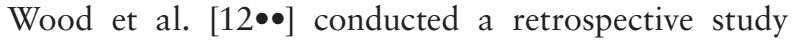
exploring the significance of Candida species isolated from BAL fluid of intensive care unit (ICU) trauma patients. This study included all patients in a trauma ICU with Candida species isolated from diagnostic BAL cultures during a 3-year period; all patients had clinical signs and symptoms of VAP, with fever, elevated leukocyte count, purulent sputum, and/or new or evolving infiltrate on chest radiograph. Of 1077 BAL cultures, 85 (8\%) grew Candida species (no colony counts $>10^{5} \mathrm{CFU} / \mathrm{mL}$ ); this was representative of a total of 64 episodes of possible Candida VAP. (For the purposes of this study, the physicians did not use 
a standard definition for Candida VAP; rather, Candida species isolated from a BAL was considered to be possible Candida VAP.) Physicians treated their patients with antifungal therapy only if their clinical suspicion was such that the presence of Candida on BAL was representative of true infection. Given these study guidelines, physicians treated only two of the 64 cases of possible Candida VAP with antifungal therapy. Despite the paucity of aggressive treatment for possible candidal VAP, no patients developed subsequent candidemia and $74 \%$ of follow-up BALs were negative for Candida species. Further, overall mortality among the patients with BAL positive for Candida species was similar to that among matched patients without Candida in respiratory tract samples.

In a more rigorous study, researchers examined 25 mechanically ventilated patients who died while in the ICU [13]. All of these patients were mechanically ventilated for more than 72 hours; none were immunosuppressed, had a diagnosis of hematologic malignancy, or were neutropenic (ANC < 1000). Twenty of 25 patients had clinically suspected pulmonary infection on the day of death; 21 of the 25 patients had pulmonary infiltrates 24 hours before death. The presence of Candida species from quantitative cultures (obtained via endotracheal aspirate, protected specimen brush, and BAL) was assessed; these findings were then correlated with microbiologic and histologic data from immediate postmortem lung biopsies (obtained via thoracotomy guided by fiberoptic bronchoscopy, and via thoracotomy by blinded approach). Forty percent of the patients (10 patients) included in this study had pulmonary biopsy cultures positive for Candida species; however, only two of these patients ( $8 \%$ of total group) had evidence of definite Candida pneumonia (one diagnosed based on histology, one diagnosed based on presence of Candida in pleural fluid culture).

How should we interpret these studies? It appears that Candida pneumonia is exceedingly rare in immunocompetent hosts. It is suspected that the isolation of Candida species from respiratory secretions might simply be the result of aspiration of colonized oropharyngeal or gastric contents [14]. At the present time, given the low frequency with which it is suspected that Candida species cause VAP in immunocompetent patients and the belief that presence of Candida species on endotracheal aspirates most often represents colonization, the current American Thoracic Society/Infectious Diseases Society of America guidelines for evaluation and treatment of VAP states that isolation of Candida species from ventilated patients "rarely requires treatment with antifungal therapy” [11].

\section{Practice patterns are not necessarily consistent with these guidelines}

Despite this evidence, many providers (in particular critical care specialists) do not feel comfortable disregarding the presence of Candida in respiratory tract samples. This has been demonstrated in several clinical studies comparing the practice patterns of infectious disease specialists with critical care specialists.

Investigators in Switzerland compared the management of suspected invasive Candida infection by infectious disease specialists and critical care specialists [15]. Sixty-five infectious disease specialists and 51 critical care specialists were presented with the following theoretical case: a 30-year-old man admitted to the ICU for multiple traumas requiring mechanical ventilation, later developing bilateral pulmonary infiltrates and fever, with BAL revealing $10^{4} \mathrm{CFU} / \mathrm{mL}$ of C. albicans (in addition to $10^{2} \mathrm{CFU} / \mathrm{mL}$ of Pseudomonas and $10^{2} \mathrm{CFU} / \mathrm{mL}$ of Acinetobacter). Of the 65 infectious disease specialists, $94.9 \%$ of them considered the Candida to be a colonizer, whereas only $46.8 \%$ of the critical care specialists interpreted it as such. Given these alternative interpretations, it was not surprising that there was also a discrepancy in the rate of prescription of antifungals: $5.1 \%$ of ID specialists versus $51 \%$ of critical care specialists would have prescribed antifungals for these patients.

\section{Should clinicians disregard the presence of Candida in respiratory tract specimens from immunocompe- tent patients?}

At the present time, there are no convincing data to suggest that empiric treatment with antifungal agents is warranted in immunocompetent patients with respiratory tract specimens revealing Candida species. With that said, however, an immunocompetent patient with positive respiratory tract specimens in addition to positive blood cultures, pleural fluid cultures, or peritoneal fluid cultures should be treated for disseminated disease. The question of treatment in an immunocompetent patient with pulmonary infiltrates and Candida recovered on BAL (without positive culture data from any other source) is less straightforward. Because the

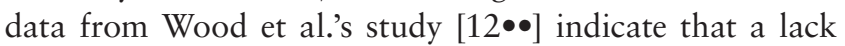
of treatment for Candida pneumonia in immunocompetent patients with clinical evidence of pneumonia and Candida on BAL does not lead to increased mortality or increased rates of candidemia, we would not recommend antifungal therapy in these patients.

Given the fact that Candida pneumonia is exceedingly rare in immunocompetent patients, investigators have sought to determine the effect of limiting the identification of Candida species in respiratory tract samples. By not providing this potentially misleading culture data, it is theorized that inappropriate initiation of antifungal therapy in these patients would be limited.

There exist interesting data to suggest that limiting the identification of Candida species in respiratory tract samples may improve patient outcomes. Barenfanger et al. [16] reported their experience with limiting identification of rapidly growing yeasts (eg, Candida species) in respiratory tract secretions (including sputum samples, BAL specimens, or PSB specimens) and the subsequent impact 
on patient, mortality, hospital stay, and cost of hospital stay. The study included 344 patients: 267 patients in a group in which yeasts were identified to the genus and/or species level (full identification group), and 77 patients in a group in which yeasts were identified as "yeasts, not Cryptococcus" (limited identification group). Of the patients included in the study, 90.1\% were immunocompetent. The study revealed that in the limited identification group, antifungal medications were used less often $(21 \%$ vs $39 \% ; P=0.004)$, patient hospital stay was shorter $(10.1$ vs 12.1 days per patient; $P=0.02)$, hospital stay costs were lower ( $\$ 6973$ vs $\$ 9407$ per patient; $P=0.03$ ), and mortality was lower (although not statistically significant at $-14.3 \%$ vs $18.7 \% ; P=0.34$ ). This argues that because of the little relevance of a respiratory tract specimen positive for Candida in an immunocompetent patient, limiting identification of the yeast may help to prevent unnecessary treatment. These results again lend credence to the idea that treatment of Candida identified in respiratory tract secretions from immunocompetent patients without clear evidence of disease is unwarranted.

\section{Identification of Candida colonization versus infection in the immunocompromised host}

If it is clinically reasonable to disregard respiratory tract samples positive for Candida in immunocompetent patients, how should we interpret respiratory tract samples positive for Candida in immunocompromised patients? This is a much less straightforward question.

It is agreed that Candida pneumonia occurs most commonly in organ transplant recipients, neutropenic patients, and in other immunocompromised hosts. Although it is more common in these patients, it is important to realize that it is still a very rare diagnosis. At the time of this writing, there were no current clinical data available to correlate the presence of positive respiratory tract specimens with clinical presentation and histologic evidence of pneumonia in today's immunocompromised patient population. However, it seems reasonable to suggest that the presence of Candida in a respiratory tract specimen from an immunocompromised patient and/or a patient with severe underlying malignancy who has clinical evidence of pneumonia be taken seriously. Although bacterial pneumonia would always be more common in such a scenario, if the patient's clinical condition deteriorates despite the use of broad-spectrum antibiotics and tissue biopsy cannot be performed, strong consideration should be given to the initiation of empiric antifungal therapy.

The gold standard for diagnosis of Candida pulmonary infection is histologic evidence of disease obtained via lung biopsy (transbronchial, thoracoscopic, or even thoracotomy approach). This constitutes an invasive approach that is not necessarily feasible to perform in all patients, particularly in those who are critically ill. Given the difficulty with identifying colonization versus infection based on respiratory tract cultures alone, there has been some interest in development of an adjunctive test to aide in the diagnosis of true invasive Candida pulmonary disease.

It has been proposed that the assessment of Candida colonization index, a measure of the burden of body sites colonized with Candida species, might be useful in the determination of risk of development of invasive infection. This has been studied predominantly in non-neutropenic patients who are critically ill. Although it appears that Candida colonization identified in multiple body sites (ie, urine, respiratory secretions, gastric secretions) or in a single body site on numerous occasions is potentially predictive of the development of future Candida infection $[17,18]$, calculation of a Candida colonization index will not aid in the definitive diagnosis of a current invasive pulmonary infection. What calculation of a Candida colonization index might do, however, is provide some guidance to clinicians regarding which patients might benefit from early empiric treatment with antifungals to prevent the development of future invasive infection.

Another potential adjunctive diagnostic test involves measurement of a serum level of $(1 \rightarrow 3)$ - $\beta$-D-glucan (BG), a polysaccharide that is a major cell wall constituent characteristic of fungi, including Candida species. Although this diagnostic test has not been studied extensively in cases of Candida pneumonia specifically, there are data to suggest that the BG assay may have high specificity and high positive predictive value in detecting invasive fungal disease that is not necessarily limited to the respiratory tract [19]. What is unclear, however, is whether this test has a high enough sensitivity when considered in the context of Candida pneumonia. As the cost of a missed diagnosis of Candida pneumonia is high, any diagnostic test relied upon should have a very high sensitivity. In the 333 patients included in the study by Ostrosky-Zeichner et al. [19], the BG assay had a sensitivity ranging from $57.1 \%$ to $79.1 \%$, depending on the BG cutoff value used.

While the BG assay is not reliable enough to rule in or rule out the diagnosis of Candida pneumonia at the present time, some investigators and clinicians speculate that use and validation of this assay in quantification of BG in BAL fluid, thereby potentially leading to an increase in the sensitivity and specificity of the diagnostic test, may serve as a valuable diagnostic adjunct in the future. There is some thought, as well, that assay of fungal metabolites such as D-arabinitol, a metabolite of several pathogenic Candida species, could ultimately play a similar role.

\section{Putting It All Together}

Candida pulmonary infection is a rare entity, but there is evidence to suggest that it does exist in very specialized patient populations. As has been described in the preceding text, the diagnosis of Candida pulmonary infection is a difficult one to make without histologic proof of disease. Given 


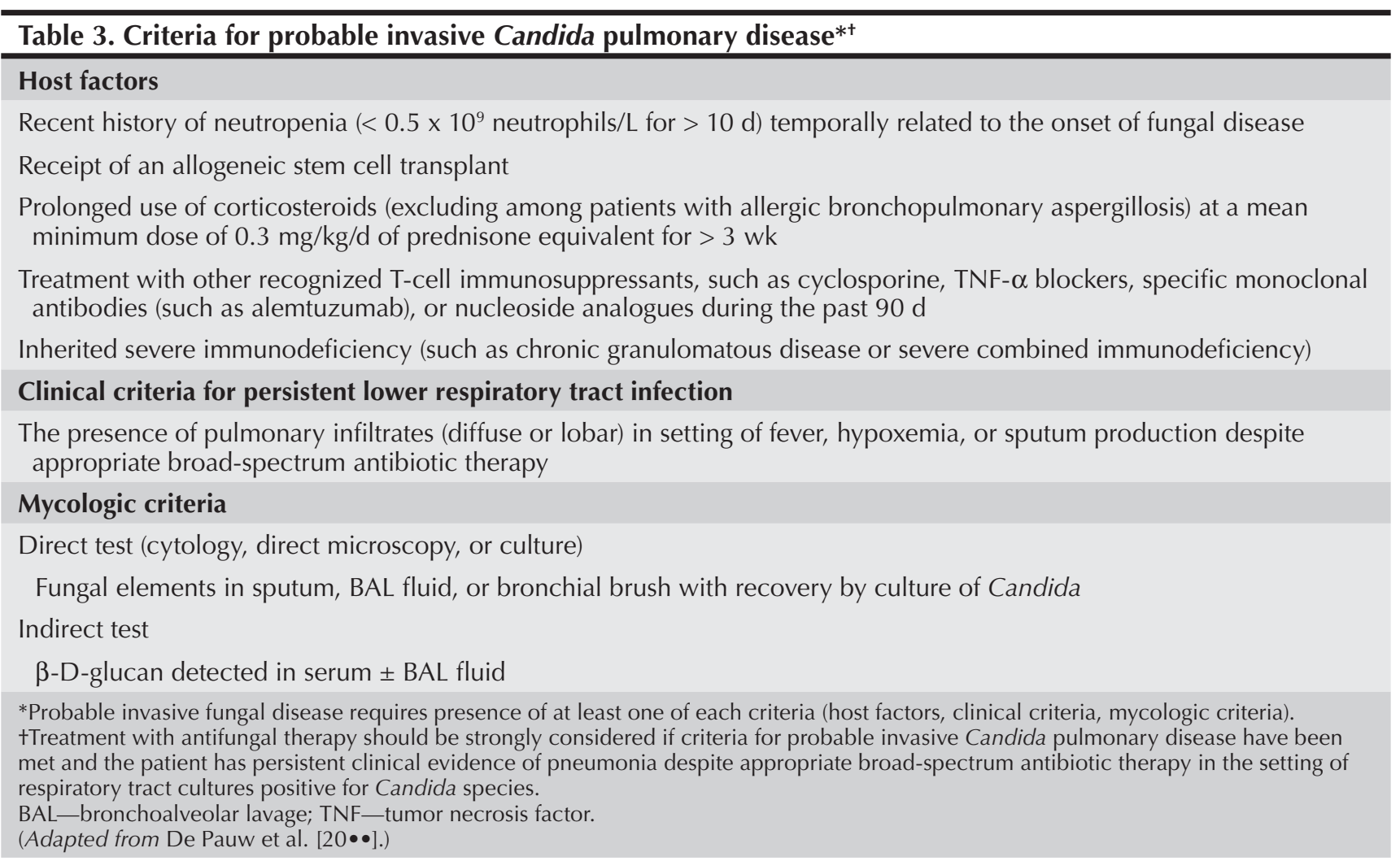

the high mortality associated with Candida pulmonary infection, however, clinicians must be able to identify those patients who are likely to have disease so that treatment can begin in an appropriate time frame. With this in mind, our recommendations for treatment of Candida pulmonary infection are described in the following text.

\section{Who should we treat?}

\section{Immunocompetent patients}

Do not treat a patient with respiratory secretion cultures positive for Candida unless there is evidence of disseminated disease (ie, positive blood cultures, pleural fluid cultures).

\section{Immunocompromised patients}

Treat if there is evidence of disseminated disease, if histology from lung biopsy (thoracoscopic, transbronchial, etc) reveals parenchymal involvement/invasion with yeasts, or if the patient has probable invasive Candida infection (Table 3) $[20 \bullet \bullet$. For example, treat if a highly immunosuppressed patient has persistent clinical evidence of pneumonia (fever, respiratory failure/insufficiency, sputum production) despite appropriate broad-spectrum antibiotic therapy in the setting of respiratory tract cultures positive for Candida species.

\section{Treatment of Candida empyema}

Because Candida empyema is still a rather rare diagnosis, rigorous data do not exist to suggest optimal management regarding antifungal therapy and drainage strategy. For this reason, it seems reasonable at the present time to apply the current guidelines regarding management of bacterial empyema, realizing that these guidelines and management concepts rely heavily upon expert opinion and are not firmly rooted in evidence-based medicine [21].

In keeping with the American College of Chest Physicians consensus statement [22] regarding medical and surgical treatment of parapneumonic effusion, any pleural effusion with positive culture/gram stain or any pleural effusion yielding pus (ie, empyema) requires drainage (either with therapeutic thoracentesis, tube thoracostomy, video-assisted thoracoscopic surgery, or surgery with thoracotomy) because of high risk of poor clinical outcome. The more difficult decision, however, involves determining which patients would benefit from surgical intervention as opposed to tube thoracostomy. Although randomized controlled trial data are lacking, the existing data suggest that of patients with large, loculated pleural empyemas, video-assisted thoracoscopic surgery is superior to chest tube drainage with regard to hospital stay and duration of chest tubes [23].

So where does this leave us regarding the management of Candida empyema? In our opinion, all Candida empyemas require drainage of some sort. However, given the fact that in our clinical experience, most Candida empyemas do not develop thick rinds and multiple loculations (as occur frequently with bacterial empyema), tube thoracostomy (kept in place until there is minimal pleural fluid output) plus systemic antifungal therapy is often adequate. 
With what should we treat?

There are three classes of drugs that are effective against Candida species. These are the azoles (fluconazole and voriconazole), echinocandins (caspofungin, micafungin, anidulafungin), and amphotericin B (both amphotericin B deoxycholate and the lipid formulations of amphotericin B). Aerosol agents like amphotericin B and lipid products of amphotericin B have been effectively used for prophylaxis in certain high-risk patients [24,25], but there are no substantial data on their use for treatment of fungal pneumonias. For treatment of Candida infection at the site of bronchial anastomosis in lung transplant patients, aerosol agents have been used successfully, but nearly always in concert with systemic antifungal therapy. Regarding systemic therapy, amphotericin B lipid complex obtains higher pulmonary tissue concentrations than liposomal amphotericin B [26], but there are no studies to show that this pharmacokinetic difference has a therapeutic advantage.

The two primary classes of drug for invasive candidiasis are azoles (fluconazole or voriconazole) and the echinocandins (caspofungin, micafungin, and anidulafungin). Both possess excellent penetration into lung tissue and fluids [27,28]. Although no specific studies in Candida pneumonia are available, it is reasonable to assume that depending on the susceptibility of the Candida species and the early institution of therapy, both classes should be effective in treatment of pulmonary candidiasis. As has been demonstrated in the setting of candidemia [29], the most important factor with regard to outcome in the setting of Candida pneumonia will likely be early diagnosis and institution of antifungal therapy.

\section{Conclusions}

Recent data have suggested that in immunocompetent patients, the presence of Candida colonization of the respiratory tract may be associated with a prolonged ICU and/or hospital stay, an increased risk of Pseudomonas VAP [30], and a potentially increased hospital mortality [31]. At the present time, however, it is unclear if the presence of Candida, in and of itself, is the potential cause of increased mortality. Rather, the presence of Candida could simply be a marker of increased host T-lymphocyte suppression, which ultimately may serve as the driving force behind increased mortality. As this story continues to unfold, it will be interesting to see if empiric therapy with antifungals in these patients will affect the described end points. If so, this could potentially alter treatment recommendations for immunocompetent patients.

\section{Disclosures}

Dr. Barkauskas reports no potential conflict of interest relevant to this article. Dr. Perfect has received research grants, honorariums, and consulting fees from Enzon, Pfizer, Merck, Astellas, Schering-Plough, and Basilea.

\section{References and Recommended Reading}

Papers of particular interest, published recently, have been highlighted as:

\section{- Of importance}

$\bullet \quad$ Of major importance

1. Masur H, Rosen PP, Armstrong D: Pulmonary disease caused by Candida species. Am J Med 1977, 63:914-925.

2. Mohsenifar Z, Chopra SK, Johnson BL, Simmons DH: Candida pneumonia: experience with 20 patients. West J Med 1979, 131:196-200.

3. Haron E, Vartivarian S, Anaissie E, et al.: Primary Candida pneumonia: experience at a large cancer center and review of the literature. Medicine (Baltimore) 1993, 72:137-142.

4. Ko SC, Chen KY, Hsueh PR, et al.: Fungal empyema thoracis: an emerging clinical entity. Chest 2000 , 117:1672-1678.

5. Kanj SS, Welty-Wolf K, Madden J, et al.: Fungal infection in lung and heart-lung transplant recipients. Report of 9 cases and review of the literature. Medicine (Baltimore) 1996, 75:142-156.

6. Chen KY, Ko SC, Hsueh PR, et al.: Pulmonary fungal infection: emphasis on microbiological spectra, patient outcome, and prognostic factors. Chest 2001, 120:177-184.

7. Schwesinger G, Junghans D, Schroder G, et al.: Candidosis and aspergillosis as autopsy findings from 1994 to 2003. Mycoses 2005, 48:176-180.

8. Roosen J, Frans E, Wilmer A, et al.: Comparison of premortem clinical diagnoses in critically ill patients and subsequent autopsy findings. Mayo Clin Proc 2000, 75:562-567.

9. Meduri GU: Diagnosis and differential diagnosis of ventilator-associated pneumonia. Clin Chest Med 1995, 16:61-93.

10. Chastre J, Fagon JY: Ventilator-associated pneumonia. Am J Respir Crit Care Med 2002, 165:867-903.

11. Guidelines for the management of adults with hospitalacquired, ventilator-associated, and healthcare-associated pneumonia. American Thoracic Society. Am J Respir Crit Care Med 2005, 171:388-416.

12.•Wood GC, Mueller EW, Croce MA, et al.: Candida sp. isolated from bronchoalveolar lavage: clinical significance in critically ill trauma patients. Intensive Care Med 2006, 32:599-603.

This study demonstrated that the presence of Candida species in BAL cultures is of limited clinical significance in immunocompetent patients who are critically ill. The majority of Candida species identified in these patients were felt to be contaminants, and despite the fact that $92 \%$ of patients did not receive antifungal therapy, no patients developed candidemia, and the overall mortality rate was similar to that observed in previous patient populations with similar degrees of injury/illness.

13. el-Ebiary M, Torres A, Fabregas N, et al.: Significance of the isolation of Candida species from respiratory samples in critically ill, non-neutropenic patients. An immediate postmortem histologic study. Am J Respir Crit Care Med 1997, 156(2 Pt 1):583-590.

14. Murray PR, Van Scoy RE, Roberts GD: Should yeasts in respiratory secretions be identified? Mayo Clin Proc 1977, 52:42-45.

15. Eggimann P, Calandra T, Fluckiger U, et al.: Invasive candidiasis: comparison of management choices by infectious disease and critical care specialists. Intensive Care Med 2005, 31:1514-1521. 
16. Barenfanger J, Arakere P, Cruz RD, et al.: Improved outcomes associated with limiting identification of Candida spp. in respiratory secretions. J Clin Microbiol 2003, 41:5645-5649.

17. Pittet D, Monod M, Suter PM, et al.: Candida colonization and subsequent infections in critically ill surgical patients. Ann Surg 1994, 220:751-758.

18. Leon C, Ruiz-Santana S, Saavedra P, et al.: A bedside scoring system ("Candida score") for early antifungal treatment in nonneutropenic critically ill patients with Candida colonization. Crit Care Med 2006, 34:730-737.

19. Ostrosky-Zeichner L, Alexander BD, Kett DH, et al.: Multicenter clinical evaluation of the $(1 \rightarrow 3)$ beta-D-glucan assay as an aid to diagnosis of fungal infections in humans. Clin Infect Dis 2005, 41:654-659.

20.• De Pauw B, Walsh TJ, Donnelly JP, et al.: Revised definitions of invasive fungal disease from the European Organization for Research and Treatment of Cancer/Invasive Fungal Infections Cooperative Group and the National Institute of Allergy and Infectious Diseases Mycoses Study Group (EORTC/MSG) Consensus Group. Clin Infect Dis 2008, 46:1813-1821.

The definition of "probable" invasive fungal disease was adapted for use in determining which patients are at high risk of developing Candida pneumonia.

21. Molnar TF: Current surgical treatment of thoracic empyema in adults. Eur J Cardiothorac Surg 2007, 32:422-430.

22. Colice GL, Curtis A, Deslauriers J, et al.: Medical and surgical treatment of parapneumonia effusions: an evidence-based guideline. Chest 2000, 18:1158-1171. [Published erratum appears in Chest 2001, 19:319.]

23. Coote N, Kay ES: Surgical versus non-surgical management of pleural empyema. Cochrane Database Syst Rev 2008, 4:CD001956.

24. Drew RH, Dodds Ashley E, Benjamin DK Jr, et al.: Comparative safety of amphotericin B lipid complex and amphotericin B deoxycholate as aerosolized antifungal prophylaxis in lung-transplant recipients. Transplantation 2004, 77:232-237.
25. Rijnders BJ, Cornelissen JJ, Slobbe L, et al.: Aerosolized liposomal amphotericin $B$ for the prevention of invasive pulmonary aspergillosis during prolonged neutropenia: a randomized, placebo-controlled trial. Clin Infect Dis 2008, 46:1401-1408.

26. Olson JA, Adler-Moore JP, Schwartz J, et al.: Comparative efficacies, toxicities, and tissue concentrations of amphotericin $B$ lipid formulations in a murine pulmonary aspergillosis model. Antimicrob Agents Chemother 2006, 50:2122-2131.

27. Fischman AJ, Alpert NM, Livni E, et al.: Pharmacokinetics of $18 \mathrm{~F}$-labeled fluconazole in healthy human subjects by positron emission tomography. Antimicrob Agents Chemother 1993, 37:1270-1277.

28. Petraintiene R, Petraitis V, Groll AH, et al.: Antifungal efficacy of caspofungin (MK-0991) in experimental pulmonary aspergillosis in persistently neutropenic rabbits: pharmacokinetics, drug disposition, and relationship to galactomannan antigenemia. Antimicrob Agents Chemother 2002, 46:12-23.

29. Garey KW, Rege M, Pai MP, et al.: Time to initiation of fluconazole therapy impacts mortality in patients with candidemia: a multi-institutional study. Clin Infect Dis 2006, 43:25-31.

30. Azoulay E, Timsit JF, Tafflet M, et al.: Candida colonization of the respiratory tract and subsequent pseudomonas ventilator-associated pneumonia. Chest 2006, 129:110-117.

31. Delisle MS, Williamson DR, Perreault MM, et al.: The clinical significance of Candida colonization of respiratory tract secretions in critically ill patients. J Crit Care 2008, 23:11-17. 